\title{
Audit: Morbidity and Mortality- Are we collecting the right data in our ENT patients?
}

Yao A, Saeed H, Mahub K, Ramamurthy L, Khwaja S. Department of Otolaryngology, Stepping Hill Hospital, Stockport NHS Foundation Trust, Poplar Grove, Hazel Grove, Stockport SK2 7JE

Stockport W/HS NHS Foundation Trust

\section{Standard}

Royal College of Surgeons Guidelines suggest the following data should be collected for discussion of cases at morbidity and mortality $(\mathrm{M}+\mathrm{M})$ meetings:

- Age

- Co-morbids

- Previous Ix (if appropriate)

- Admitting diagnosis

- MDT decisions (if appropriate)

- Operation

- Complication

- Details of events around the complication.

Collection of such data is integral to promoting improvement in surgical safety, quality and learning.

\section{Data Collection}

Data was collected retrospectively from the last 6 months from data sources including: $M+M$ meeting records, $M+M$ data collection templates, $\mathrm{M}+\mathrm{M}$ powerpoint presentations.

\section{Inclusion criteria:}

Intra-operative complications

Surgical inpatient deaths

Returns to theatre.

Never events

Patients whose discharge was delayed by complications.

Readmissions $<30$ days since discharge.

Other "major importance"

Other near misses

\section{Analysis}

26 patients were included. 4 patients were excluded as these were complications from operations done at other hospitals. Poor recording of co-morbidities was noted $(9.1 \%)$. But this is important to record as hypertension and anti-coagulants are significant risk factors to the common complication of bleeding.

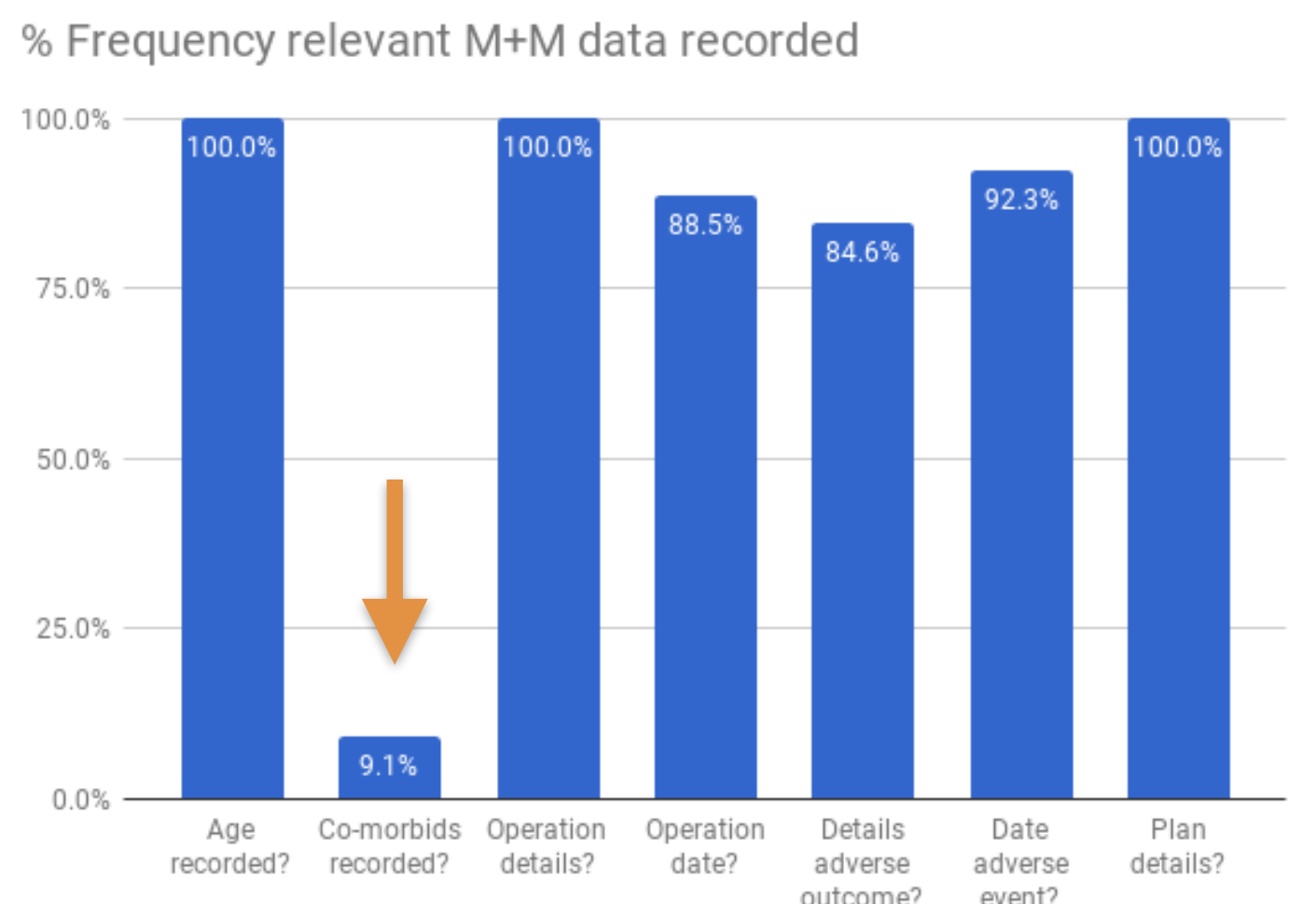

\section{Intervention: Data Collection Tool}

We created an updated data collection tool using an Excel spreadsheet. This contained additional and compulsory columns: co-morbids, operation date, details of adverse event (and grading), and details of management plan.

\section{4a. Excel data collection tool:}

\begin{tabular}{|c|c|c|c|c|}
\hline A & B & C & D & $\mathrm{E}$ \\
\hline Number & Month entered & Sex & AGE (YRS) & Co-morbidi \\
\hline \multicolumn{5}{|c|}{ EXAMPLE January } \\
\hline \multicolumn{5}{|l|}{1} \\
\hline \multicolumn{5}{|c|}{ February } \\
\hline \multicolumn{5}{|l|}{2} \\
\hline \multicolumn{5}{|l|}{3} \\
\hline \multicolumn{5}{|l|}{$\mathbf{F}$} \\
\hline \multicolumn{5}{|c|}{ OPERATIVE / ADMISSION DETAILS } \\
\hline PROCEDURE & INDICATION & DATE & Primary surgeon & CONSULTANT \\
\hline K & L & M & $\mathbf{N}$ & $\mathbf{P}$ \\
\hline \multicolumn{5}{|c|}{ COMPLICATION 1} \\
\hline COMPLICATION & \begin{tabular}{|l|l|} 
& DETAILS \\
\end{tabular} & GRADE & STIFIED & PLAN \\
\hline
\end{tabular}

\section{4b. Keys to the data collection tool we used:}

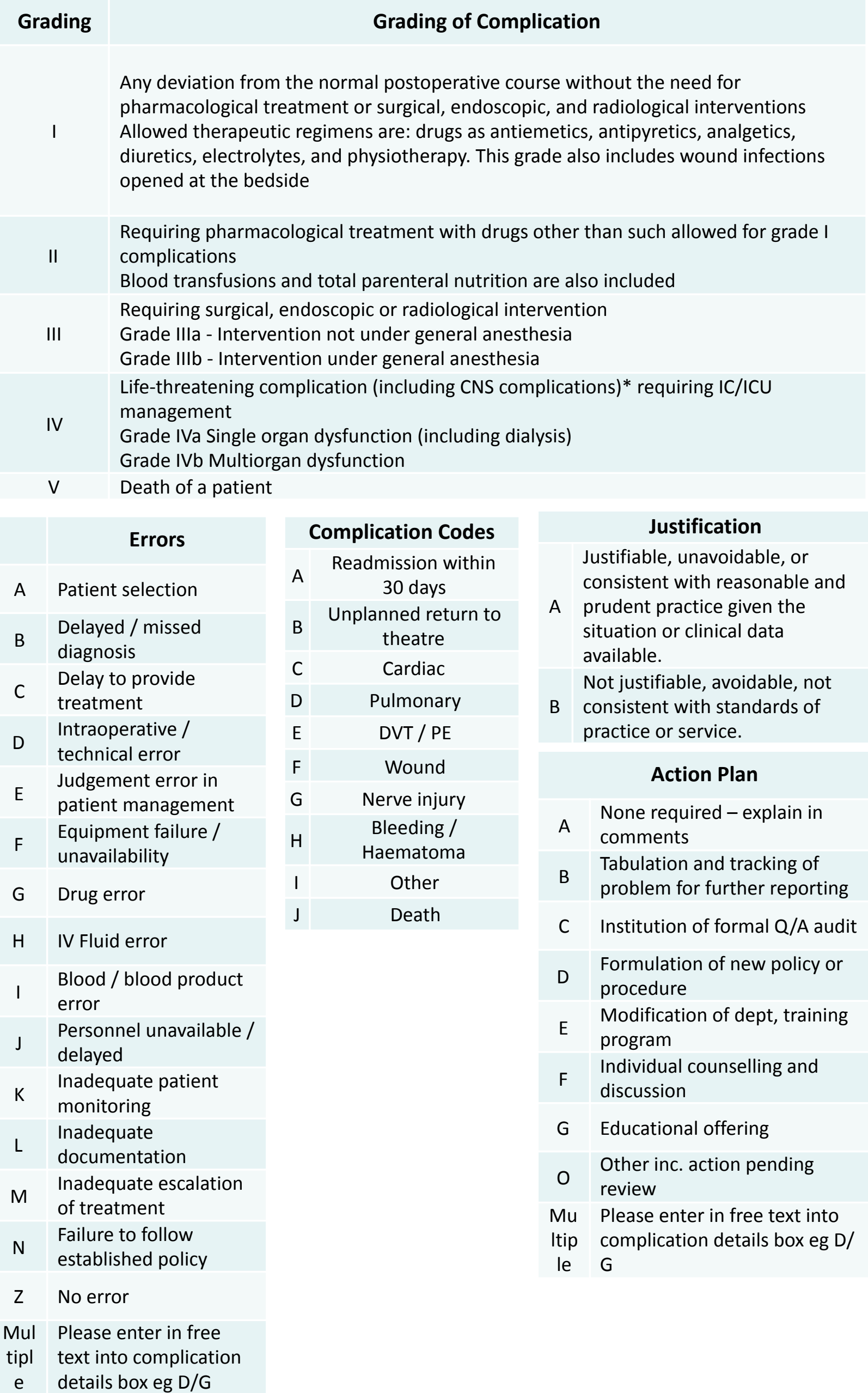

